\title{
Urgences
}

\section{Le conflit des poétiques dans Beauté baroque}

\section{François Dumont}

Numéro 28, mai 1990

Le roman comme poétique

URI : https://id.erudit.org/iderudit/025588ar

DOI : https://doi.org/10.7202/025588ar

Aller au sommaire du numéro

Éditeur(s)

Urgences

ISSN

0226-9554 (imprimé)

1927-3924 (numérique)

Découvrir la revue

Citer cet article

Dumont, F. (1990). Le conflit des poétiques dans Beauté baroque. Urgences, (28), 48-55. https://doi.org/10.7202/025588ar d'utilisation que vous pouvez consulter en ligne.

https://apropos.erudit.org/fr/usagers/politique-dutilisation/ 


\section{Le conflit des poétiques dans Beauté baroque}

François Dumont

Lire Claude Gauvreau, c'est tenir dans ses mains un énorme volume, publié six ans après le suicide de l'auteur, et qui se présente comme son unique livre ${ }^{1}$. Celui-ci, par son allure d'« œuvre complète" irréductible, par le mythe qui l'entoure, appelle, au premier abord, soit une adhésion totale, soit un rejet aveugle; il est en quelque sorte « lu d'avance * et continue ainsi, une fois publié, de se dérober à la lecture.

Deux pistes de lecture sont toutefois proposées par la structure même du livre: la datation, souvent très précise, et la répartition des œuvres en genres. Par la datation, chaque œuvre est liée à celle qui précède et à celle qui suit; le livre de Gauvreau se présente comme un déploiement continu et même, j'y reviendrai, comme la succession d'« acquis" dans un mouvement de "progrès". Parmi la multitude des genres (théâtre, poèmes, opéra, tragédie, "téléthéâtre cosmique", etc.), on trouve un seul roman: Beauté baroque, daté de 1952. Cette unicité, ainsi que la situation déterminante du roman dans la succession des œuvres ${ }^{2}$, justifie qu'on accorde une attention particulière à Beauté baroque. En outre, pour aborder l'immensité des CEuvres créatrices completes, il est éclairant de distinguer les œuvres et, plus fondamentalement, les poétiques. S'il est vrai, par exemple, que les premiers poèmes de Gauvreau prolongent dans des directions originales la poétique surréaliste, d'autres œuvres, loin de la prolonger, la nient. C'est le cas, me semble-t-il, de Beauté baroque.

1 Claude Gauvreau, CEuvres créatrices complètes, Montréal, Parti pris, 1977, $1503 \mathrm{p}$. Les autres publications de Gauvreau sous forme de livre (trois plaquettes: Brochuges, Étal mixte et Sur fil métamorphose) sont reprises dans cette édition. Par ailleurs, l'auteur a ecarté plusieurs textes de ces « œuvres complètes", notamment ses écrits théoriques et polémiques, ainsi que de nombreux textes pour la radio.

2 Dans Claude Gauvreau, poète et mythocrate (Montréal, VLB éditeur, 1979), Jacques Marchand divise l'itinéraire de Claude gauvreau en deux parties: "l'encre rouge ", période d'expérimentation, et a l'encre noire", période de mystification. La coupure s'effectuerait précisément avec Beauté baroque. II en sera question plus loin. 
En guise d'introduction aux Euvres creatrices completes, on peut lire, à la suite d'un bref *Avertissement* de l'éditeur Gérald Godin, une "Autobiographie". Après avoir évoqué le suicide de la comédienne Muriel Guilbault, «muse incomparable de [sa] vie» (p. 12), Gauvreau y présente son roman de la façon suivante: «Le cadavre de Muriel ayant été souillé par d'abjects moralisateurs de diverses disciplines, je me décidai à laver sans réplique possible cette ignominie en écrivant le roman de sa vie tel que je la connaissais » (p. 13). « Laver sans réplique possible», qu'est-ce à dire? Gauvreau s'en est expliqué ailleurs: «Mon obsession était de gagner le prix du Cercle du Livre de France, afin de faire triompher le souvenir de Muriel que certaines goujateries avaient odieusement maculé ${ }^{3}$. C'est donc dans le but de faire reconnaitre son roman comme $*$ chefd'œuvre" qu'il le soumet au concours du Cercle du Livre de France (remporté cette année-là par André Langevin pour Poussière sur la ville). D'autre part, la formule «le roman de sa vie tel que je la connaissais " établit un "pacte biographique" qui associe le roman au témoignage plutôt qu'à la fiction.

Par ce dernier aspect, le roman de Gauvreau s'accorde avec la poétique surréaliste du roman, selon laquelle le récit " reçu * s'oppose à la fiction «fabriquée». De plus, on ne trouve nulle trace de description ou de dialogue, procédés condamnés par Breton dans le premier Manifeste du surréalisme. Mais Gauvreau, qui affiche explicitement Nadja en conclusion à son roman ${ }^{4}$ et qui se reprochera de ne pas avoir envoyé son manuscrit à Breton ${ }^{5}$, contredit pourtant absolument l'esprit du surréalisme, d'abord, bien sûr, en assumant la notion de « chefd'œuvre ", mais encore, et surtout, en recourant continuellement aux «lois de la psychologie* (p. 459). C'est précisément la "psychologie toute faite" que les surréalistes ont toujours

3 Claude Gauvreau, L'épopé automatiste vue par un cyclope ", La barre du jour, no 17-20, janvier-aout 1969, p. 92.

4 Adieu, blancheur de la neige!

Adieu ineffable!

Aurélia. Eurydice. Nadja. "

Beauté baroque, dans Euvres créatrices complètes, op. cit., p. 500. Le roman couvre les pages 379 a 500 .

5 Je me souviens que [Jean-Paul] Mousseau me conseilla d'envoyer le roman a André Breton, car c'était "trop fort pour le Cercle". Fernande [SaintMartin], elle, me conseilla d'inscrire Beauté baroque au concours du Cercle. [...] Naturellement, c'est le vieux Mousseau qui avait raison " ( « L'épopée automatiste vue par un cyclope ", op. cit., p. 92). 
50

reprochée au genre romanesque, dans la mesure où elle assujettit l'inconscient à la raison ${ }^{6}$. Dans Beauté baroque, le psychologisme péremptoire du narrateur est omniprésent. Ses manifestations vont du plus convenu - * Par intérim, ils allaient tous payer pour son père (p. 388) - au plus contourné: * Les enfants abhorrent ceux qui les détestent: pourtant, paradoxe infamant, ils se sentent coupables de la laideur qu'on leur prête. L'enfance ressuscitée ressuscitait la convenance d'expier la désaffection subie" (p. 399). Beauté baroque est saturé de lois et de maximes. Le roman, pour Gauvreau, est d'abord une explication: la vie de la *beauté baroque n est un destin que le narrateur relie toujours à des causes. Une poétique du. roman, plus près du roman psychologique que du surréalisme, est donc implicite dans Beauté baroque (on pourrait aussi, sans doute, induire une "poétique du chef-d'œuvre»). Mais c'est la poétique de la poésie qui en est le véritable enjeu.

Explication et justification d'une existence, Beauté baroque est aussi la célébration d'une femme et, par une identification systématiquement entretenue dans le récit, de la poésie: «c'était une poésie vivante que cet être* (p. 393), *artiste-poète" (p. 418), * poétesse irréductible* (p. 470), " précurseur de la poésie régnante* (p. 497). Autant la femme est à la fois individu et actrice, autant la poésie correspond, au début du roman, à la *Vie » comme aux *Lettres * : « Ce que les grands poètes vivent en l'imaginaire, elle avait la puissance de l'imposer au quotidien (p. 392). Ainsi le *génie» de la femme fait-il coïncider la *furie des viscères * avec la *lettre» d'une tragédie classique:

A quinze ans, elle avait appris par cour les cinq actes d'une tragédie.

Elle ne connaissait pas les règles de la versification. Elle ne connaissait, à merveille, que la justesse naturelle d'une compréhension précise.

Elle avait débité, dans la furie sacrée des viscères possédés, la lettre de tout ce qu'elle savait. Les attentifs comprirent: la salive du génie s'étalait devant eux.

6 Sur la poétique surréaliste du roman comme tentative de a déjouer " le romanesque, voir l'essai de Jacqueline Chénieux, Le surréalisme et le roman, Lausanne, L'âge d'homme, 1983 (cf. particulièrement, autour de Nadja, p. 171177). 
Un classique du courage compétent: ainsi sa brave exhibition s'avéra. (p. 384)

Deux coïncidences sont donc posées: coïncidence de la femme avec la poésie; coïncidence, dans la poésie, de la "Vie " et des «Lettres *. La première n'est jamais mise en cause. C'est la seconde qui pose problème. Et d'une façon très différente, encore une fois, de la dynamique surréaliste.

Dans le surréalisme, la poésie entretient des rapports non seulement problématiques, mais carrément hostiles avec les «Lettres ». La poésie intéresse les surréalistes en dehors et en dépit des cadres esthétiques et institutionnels littéraires: * la poésie, qui est tout ce qui m'a jamais souri dans la littérature", affirme André Breton, «émane davantage de la vie des hommes, écrivains ou non, que de ce qu'ils ont écrit ou de ce qu'on suppose qu'ils pouvaient écrire». Il ajoute, parlant de certains poètesphares du surréalisme: " leur attitude en tant qu'hommes laisse loin leurs mérites d'écrivains et [...] seule cette attitude donne un sens véritable à leur œuvre, telle que nous l'admirons » ${ }^{7}$. Il en va tout autrement dans Beauté baroque. La poésie qui, à l'origine, s'accordait avec la "Vie", devient strictement « littéraire *, et il en est ainsi pour le roman lui-même. L'écriture devient un combat entre différentes tendances à l'intérieur des Lettres. C'est ainsi que Courteline est confronté à Nerval (p. 391) et que le narrateur se mue en nouveau "Valjean " (p. 425). Le titre de Beauté baroque ne désigne plus une femme mais un livre. Gauvreau écrit: "Entre elle et mon projet s'improvisa, en quelque sorte, un coup de foudre" (p. 412). Or, il s'agit moins d'un coup de foudre que d'une substitution ${ }^{8}$. La "Vie* est abandonnée; la « femmepoésie» se transforme en «femme-chef-d'œuvre»:

Les chefs-d'œuvre de l'art n'ont pas de chair tiède à sacrifier à nos palpations: certain donnerait sa vie pour sauver de la destruction quelques-uns. La jeune femme, pour moi, avait cessé d'être essentiellement une humanité à convoiter: je la voyais un chef-d'œuvre à contempler, dont je devenais le conservateur consciencieux.

$7 \quad$ André Breton, Les pas perdus, Paris, N.R.F., 1924, p. 134 et 137.

8 Soulignons, à cet égard, l'explication que le narrateur-psychologue donne du suicide de l'actrice: elle subit un avortement dans sa jeunesse, à la suite duquel elle devint stérile, d'où son désespoir. La solution avancée par le narrateur, on le devine, est de confier à l'art ce que la vie refuse: * Un métier passionnant eut pu devenir sa justification, l'enfant de son cerveau. " (p. 407) 
52

Elle ne perdait rien de sa matière de femme. Sa chair, inusable et royal don, demeurait à offrir : encore elle donnerait sa chair, à moi-même... Seulement, ce n'était plus là sa fonction principale d'objet: analogue à l'inestimable couvre d'art, son utilité primordiale devenait de nourrir la contemplation. (p. 461)

Il y aurait beaucoup à dire, évidemment, sur la conception de la femme que cet extrait révèle, et notamment sur les correspondances possibles entre la louange et le mépris. Je ne retiens ici que la conception que le narrateur se fait de l'œuvre: un objet, une chose indépendante de toute humanité. Ici la poésie donne, littéralement, sa vie. Le matérialisme affiché de Gauvreau, ce qu'il appelait "monisme athée», résulte peut-être moins de l'opposition à la transcendance que de la défaite d'une poétique fondée sur * La vie qui nous donne la manne de poésie» (p. 415). De ce point de vue, le sous-titre de Beauté baroque, «roman moniste», s'il veut marquer une caractérisation philosophique, indique également un passage. Du baroque, lieu du conflit - car * Le juste milieu n'est pas l'affaire du baroque * (p. 478) - le narrateur passe au monisme d'*objets sortis de [sa] personne et qui sont parvenus à une réalité autonome» (p. 447). La « mort du référent * prend ici la forme d'un deuil - et non d'une dénonciation comme dans le Nouveau Roman -, ce qui éclaire la nature du langage que Gauvreau qualifie d'، exploréen $*$.

Dans ses textes théoriques, Gauvreau distingue quatre types d'images: «l'image rythmique, l'image mémorante, l'image transfigurante, l'image exploréenne*, types constituant une progression vers « Des bribes de mots abstraits connus, modelés dans une intrépide sarabande inconsciente $*$. L'exploréen, ainsi défini comme éclatement du sens, est au cœur de la poétique de Claude Gauvreau: «je ne connais pas de textes à accent définitivement exploréen autres que les miens», affirme-t-il. «Dans mes textes, le côtoiement constant et l'entrelacement des mots quotidiens et des mots exploréens est une des causes les plus fréquentes de la stupéfaction des lecteurs. C'est aussi la caractéristique la plus marquée de ma patte ${ }^{9}$.

9 Claude Gauvreau, "Lettre à un fantôme ", La barre du jour, n ${ }^{\text {s }}$ 17-20, janvier-aout 1969, p. 349,357 et 360. 
À première vue, Beauté baroque ne pose pas la question habituelle du néologisme chez Gauvreau. Ce roman, en effet, est l'une des rares cuvres de l'auteur où le langage exploréen n'est pas utilisé. Un épisode du roman, toutefois, peut être lu comme une mise en abyme de la poétique exploréenne dans l'œuvre de Gauvreau. Ce passage, qui précède immédiatement la transformation de la « femme-poésie * en «femmechef-d'œuvre», est la relation d'une crise du narrateur qui monte sur le toit lorsqu'il comprend que jamais il ne sera l'amant de la "beauté baroque»:

Faux suicide? Peut-être.

Je gesticule, je profère des paroles inintelligibles. A un pouce du vide. Gestes grandiloquents, tonnerre de sons verbaux: par une mise en scène dans l'espace, je déverse le surplus d'intolérable. Je ne parviens pas à épuiser, à pulvériser ma souffrance: je parviens cependant à rendre ses proportions conciliables à l'existence.

Je redeviens apte au raisonnable. Par cette saignée pitoyable, les gonflements putréfiants ont été expulsés de mon corps, je retourne à une normalité viable.

Vidé de mes secousses apeurantes, bizarrement soumis, dégonflé, un peu tragique, je rentre dans l'appartement. Je tremble. C'est fini. (p. 458)

Dans la mesure où les * paroles inintelligibles " sont assimilables au langage exploréen, celui-ci naît d'une exacerbation émotive, bientôt conjurée par une "mise en scène * permettant le retour à la normalité. Gauvreau aimait à répéter que "la conséquence est plus importante que le but *, et c'est selon ce principe que, dans ses textes théoriques, il insiste sur l'origine du langage exploréen ( Commotion de volcan émotif "10), sans assigner de but à sa poétique, sinon la "libération du désir». Dans le roman, la *conséquence» est un retour à la normalité, et il n'est pas sans intérêt de remarquer qu'à la faveur de ce retour, le narrateur fétichise la * femmepoésie ", la transformant en objet littéraire, et se transformant lui-même en «homme de lettres ».

Il me paraît pertinent de rapprocher cette transformation et l'état du groupe automatiste à l'époque où Gauvreau écrit 
54

son roman. Tout juste après la rédaction de Beauté baroque, Gauvreau réussit, non sans difficultés, à réunir une dernière fois le groupe automatiste pour l'exposition La matiere chante, véritable chant du cygne. Après cette exposition, le groupe se disloquera définitivement, et les préoccupations sociales des signataires de Refus global feront désormais place à des préoccupations davantage esthétiques. Ainsi la rhétorique du *progrès social " cède le pas, chez le polémiste Gauvreau, à une rhétorique du "progrès esthétique *: la révolution dont parle abondamment Gauvreau dans les années soixante devient essentiellement littéraire ${ }^{11}$.

Voilà qui correspond, d'une certaine façon, à la lecture de Jacques Marchand dans Claude Gauvreau, poete et mythocrate. Selon Marchand, à partir de Beauté baroque, l'œuvre de Gauvreau se transforme radicalement: le poète passe d'une poétique novatrice post-surréaliste à une poétique mystificatrice, fondée sur un "progrès littéraire" strictement «livresque 12 . Le langage exploréen n'est plus alors le résultat d'une forme originale d'écriture automatique, mais l'émaillage savamment dosé de " trouvailles ».

Je ne crois pas, pour ma part, que l'itinéraire de Gauvreau soit aussi linéaire. Sans doute Gauvreau s'éloigne-t-il progressivement du surréalisme comme esprit et comme méthode. Mais la tension entre la «Vie * et les * Lettres * me semble traverser son œuvre entière: le conflit des poétiques prend des formes différentes selon les œuvres et, dans les meilleurs des cas, ce conflit dynamise les textes. Il est vrai que dans Beauté baroque, le conflit étant résolu, la figure du "chef-d'œuvre règne statiquement sur le roman. Tel n'est pas le cas dans les premiers textes de Gauvreau, où *le plus grand des poètes" (p. 19) est confronté à une jeune fille qui revendique "La vie! La vie!» (p. 28). Par ailleurs, dans les pièces de thêatre les mieux connues de Gauvreau - La charge de l'orignal épormyable et Les oranges sont vertes - le langage exploréen est à la fois glossolalie et expression du "génie littéraire», et cette dualité contribue à donner au discours sur la folie sa consistance.

$11 \propto[\ldots]$ la révolution automatiste (non figurative) n'a jamais eu lieu en poésie; cette absence révolutionnaire étant imputable a la répression quasi totale qu'a subie mon couvre. " (Claude Gauvreau, "Commentaire thérique ", dans Guy Robert, Litterature du Québec. Poésie actuelle, Montréal, Déom, 1970, p. 99).

12 Jacques Marchand, Claude Gauvreau, poete et mythocrate, op. cit., p. 269. 
Le conflit des poétiques n'est sûrement pas la seule source de dynamisme dans l'œuvre de Gauvreau. Mais il constitue une voie d'entrée invitante dans un univers volontiers hostile. En outre, à partir des manifestations de ce conflit dans Beauté baroque, il est possible de dégager certains rapports de Gauvreau au surréalisme, abstraction faite du discours théorique de l'auteur, discours qu'il a, d'ailleurs, luimême exclu de ses «œuvres créatrices".

Il serait absurde d'intenter à Gauvreau un * procès de surréalisme » et de fonder là-dessus - ou sur un éventuel "dépassement» - la valeur de son œuvre. Mais il est clair que, malgré la filiation, l'écriture de Gauvreau, dans ses fondements mêmes, differre notablement du texte surréaliste. Pour ce qui est du roman, en particulier, le psychologisme appuyé et la participation déclarée de Beauté baroque à la Littérature s'opposent à la poétique surréaliste ${ }^{13}$. En fait, dans Beauté baroque comme dans plusieurs œuvres de Gauvreau, le texte procède moins du surréalisme qu'il ne le thématise. Gauvreau qui, dans certaines de ses pièces, met en scène ses amis automatistes, met aussi en scène l'automatisme lui-même: ses contradictions et son désir de réconcilier l'art et la vie.

13 Ce qui n'exclut pas, bien entendu, certains rapprochements, notamment du coté de la stylistique. Voir à ce sujet les articles de Réal Ouellet et Hélène Vachon, * La célébration de l'exces *, Lettres québécoises, $n^{\circ} 7$, aout-septembre 1977, p. 19-21, et d'André-G. Bourassa, * La seule histoire d'amour », Livres et auteurs québécois 1977, Québec, P.U.L., 1978, p. 311-314. 